PROCEEDINGS OF THE

AMERICAN MATHEMATICAL SOCIETY

Volume 126, Number 5, May 1998, Pages 1541-1545

S 0002-9939(98)04287-7

\title{
IMPROVING THE METRIC IN AN OPEN MANIFOLD WITH NONNEGATIVE CURVATURE
}

\author{
LUIS GUIJARRO
}

(Communicated by Christopher Croke)

\begin{abstract}
The soul theorem states that any open Riemannian manifold $(M, g)$ with nonnegative sectional curvature contains a totally geodesic compact submanifold $S$ such that $M$ is diffeomorphic to the normal bundle of $S$. In this paper we show how to modify $g$ into a new metric $g^{\prime}$ so that:

1. $g^{\prime}$ has nonnegative sectional curvature and soul $S$.

2. The normal exponential map of $S$ is a diffeomorphism.

3. $\left(M, g^{\prime}\right)$ splits as a product outside of a compact set.

As a corollary we obtain that any such $M$ is diffeomorphic to the interior of a convex set in a compact manifold with nonnegative sectional curvature.
\end{abstract}

\section{INTRODUCTION}

The existence of a metric with nonnegative curvature in an open Riemannian manifold sets important restrictions on its structure. For example, they contain a compact, totally geodesic submanifold $S$, called the soul, so that the total space is diffeomorphic to its normal bundle $\nu(S)$. In this note we study how, given such a metric, we can construct another one satisfying stronger conditions. In particular, we prove the following result:

Theorem A. Let $(M, g)$ be a compact convex manifold with smooth boundary, nonnegative sectional curvature and soul $S$. Then there exists a complete metric $\bar{g}$ in $M$ with nonnegative curvature and that splits as a metric product $N \times[a, \infty)$ outside some tubular neighborhood of $S$, where $N$ is diffeomorphic to the unit normal bundle of $S$ in $M$.

The following two facts are easy consequences:

Theorem 1.1. Let $M$ be an open manifold with nonnegative sectional curvature and soul $S$. Then there is a (possibly different) metric in $M$ with nonnegative curvature, soul $S$ and whose normal exponential map $\exp : \nu(S) \rightarrow M$ is a diffeomorphism. Moreover, the ideal boundary $M(\infty)$ of $M$ with the new metric is only a point.

Theorem 1.2. Let $M$ be an open manifold with nonnegative sectional curvature and soul $S$. If $D(S)$ is the normal disc bundle of $S$ in $M$, then there is a metric with nonnegative sectional curvature in the double of $D(S)$.

Received by the editors October 25, 1996.

1991 Mathematics Subject Classification. Primary 53C20.

(C)1998 American Mathematical Society 
Recall that the double of $D(S)$ is the space obtained after gluing together two copies of $D(S)$ by the identity map of the boundary. Theorem 1.1 could help determine which vector bundles admit metrics with nonnegative curvature, since it allows us to assume that such metrics are of a rather simple form. Theorem 1.2 should help in constructing new compact manifolds with nonnegative curvature.

The proof of Theorem A uses some consequences of the main theorem in [5] that were indicated to us by G. Walschap, as well as a simple modification of a construction due to Kronwith ([3]).

The author would like to thank Gerard Walschap for telling us about Lemma 2.2, Karsten Grove for pointing out Remark 4.3, and Vitali Kapovitch, Kris Tapp, Wolfgang Ziller and the referee for a careful reading of a preliminary version of this paper and helpful suggestions.

\section{Convexity around the soul}

Let $M$ be a manifold with nonnegative sectional curvature and (possibly empty) smooth convex boundary. Recall that we denote its soul by $S$ and its normal bundle by $\nu(S)$. There exists a distance-nonincreasing retraction $s h: M \rightarrow S$, used by Perelman to prove the following result:

Theorem 2.1 (Perelman's rigidity theorem [5]). 1. Let $\alpha:[0, \infty] \rightarrow S$ be a geodesic, and $u \in \nu_{p}(S)$ a normal vector to the soul. Let $U$ be the vector field along $\alpha$ obtained by parallel transport of $u$. Then

$$
R(t, s)=\exp _{\alpha(t)} s U(t), \quad t \in[0, a], s \in[0, \infty),
$$

is a flat rectangle totally geodesically immersed in $M$.

2. $\operatorname{sh}\left(\exp _{p} t u\right)=p$ for all $t \in[0, \infty)$.

3. sh: $M \rightarrow S$ is a $C^{1}$ Riemannian submersion.

It follows from this that $s h$ is $C^{\infty}$ in a tubular neighborhood of $S$. We will use $\mathcal{H}$ and $\mathcal{V}$ for the horizontal and vertical distributions associated to this submersion.

We denote by $D_{r}$ the set of points in $M$ at distance not greater than $r$ from $S$. When the soul is a point, $D_{r}$ is convex for small $r$. It was pointed out to us by G. Walschap that Theorem 2.1 extends this to the case of nontrivial souls:

Lemma 2.2. $D_{r}$ is convex for small $r>0$

The result can be proved as in [2], Lemma 1.2, part (a), which only differs from Lemma 2.2 in that it includes an extra hypothesis that follows from Perelman's theorem.

\section{Model Metrics in tubular neighborhoods of The SOUL}

In this section we will adapt the construction of [3] to prove Theorem A.

Let $M, S$ be as in Theorem A and let $\nu_{1}(S)$ denote the unit normal bundle of the soul in $M$. By rescaling, we can always assume that exp $: \nu_{1}(S) \times(0,10) \rightarrow M$ is injective, sh: $D_{10} \rightarrow S$ is smooth, and $D_{r}$ is convex for $r \leq 10$.

Define $\tilde{\chi}: D_{2} \rightarrow \mathbb{R}$ as

$$
\tilde{\chi}(x)= \begin{cases}1 / d\left(x, \partial D_{2}\right), & x \in D_{2}-D_{\frac{1}{2}}, \\ \chi(x), & x \in D_{\frac{1}{2}},\end{cases}
$$

where $\chi$ is any smooth extension of $d\left(x, \partial D_{2}\right)^{-1}$ to $D_{\frac{1}{2}}$ with $0 \leq \chi \leq \frac{2}{3}$. 
Choose also a real function $g:(-\infty, 1] \rightarrow \mathbb{R}$ so that:

- $g$ is smooth in $(-\infty, 1)$ and continuous at 1 .

- $g=0$ on $\left(-\infty, \frac{2}{3}\right), g(1)=1$.

- $g^{\prime}$ and $g^{\prime \prime}$ are positive on $\left(\frac{2}{3}, 1\right)$.

- $g^{-1}:[0,1] \rightarrow\left[\frac{2}{3}, 1\right]$ has all left derivatives zero at 1 .

The last condition assures that

$$
G(y)= \begin{cases}g^{-1}(y) & \text { if } y \in[0,1] \\ 1 & \text { if } y>1\end{cases}
$$

is a smooth function, which is basically the only modification done to the argument of [3].

Finally, we define $\Gamma: D_{1} \rightarrow \mathbb{R}$ by $\Gamma=g \circ \tilde{\chi}$.

For $P=D_{2} \times \mathbb{R}$, consider the subset $L=A \cup B$, where

$$
\begin{aligned}
& A=\text { Graph } \Gamma=\left\{(x, \Gamma(x)): x \in D_{1}\right\}, \\
& B=\partial D_{1} \times[1, \infty) .
\end{aligned}
$$

Obviously, $A \cap B=\partial D_{1} \times\{1\}$.

Lemma 3.1. $L$ is a smooth convex hypersurface of $P$.

Proof. To prove smoothness of $A \cap B$, define $\phi: \nu_{1}(S) \times\left(\frac{1}{2}, \frac{3}{2}\right) \rightarrow P$ as

$$
\phi(u, s)=\left(\gamma_{u}\left[2-\frac{1}{G(s)}\right], s\right) .
$$

Its image is clearly a neighborhood of $A \cap B$ in $L$. Furthermore, since the set of vectors tangent to curves of the form $\phi(u(t), 1)$ and $\phi(u, s)$ generate $T_{\phi(u, 1)} L, \phi$ is an immersion.

For the convexity part, recall that for $p \in D_{2}$ we have an orthogonal decomposition

$$
T_{p} D_{2}=\mathcal{H}_{p} \oplus \mathcal{V}_{p}=\mathcal{H}_{p} \oplus \widetilde{\mathcal{V}}_{p} \oplus \mathbb{R} \gamma^{\prime},
$$

where $\gamma$ is the unique minimizing geodesic joining the soul to $p$. This induces a decomposition of $T L$ of the form

$$
T_{(p, \Gamma(p))} L=\mathcal{H}_{p} \oplus \widetilde{\mathcal{V}}_{p} \oplus \mathbb{R}\left\{\gamma^{\prime}(t)+(\Gamma \circ \gamma)^{\prime}(t) \partial_{t}\right\}
$$

for $(p, \Gamma(p)) \in A$ with $p=\gamma(t)$, and

$$
T_{(p, s)} L=\mathcal{H}_{p} \oplus \widetilde{\mathcal{V}}_{p} \oplus \mathbb{R} \partial_{s}
$$

for $(p, s) \in B$.

Suppose then that $(p, \Gamma(p)) \in A$ with $p=\gamma(t)$, where $t<1$. For $F: P \rightarrow \mathbb{R}$ defined as $F(x, t)=t-\Gamma(x)$, we have $A=F^{-1}(0)$. Thus its second fundamental form is proportional to Hess $\Gamma$. A standard computation (see [3]) shows that

$$
\text { Hess } \Gamma(v, v)=\left(g^{\prime} \circ \tilde{\chi}\right) \operatorname{Hess} \tilde{\chi}(v, v)+\left(g^{\prime \prime} \circ \tilde{\chi}\right)(v(\tilde{\chi}))^{2} .
$$

This is nonnegative because of the choice of the function $g$ and the fact that $d\left(x, \partial D_{2}\right)$ is convex in $D_{2}[1]$.

On the other hand, if $(p, s) \in B, s>1$, then

$$
\nabla_{\partial_{s}}^{P} \gamma^{\prime}=0, \quad \nabla_{x}^{P} \gamma^{\prime}=\nabla_{x}^{M} \gamma^{\prime}
$$

Since we chose $D_{1}$ to be convex, $\partial D_{1}$ has a positive semidefinite second fundamental form, and thus the same is true for $B$. By continuity, this is also valid for $A \cap B$. 
Theorem A is now an easy consequence of Lemma 3.1 and the Gauss formula.

Remark 3.2. The metric in $\partial D_{1}$ at $B$ is the same it receives from $M$. This is clear because the embedding of $D_{1}$ in $P$ as the graph of $\Gamma$ only modifies the original metric of $D_{1}$ in the radial direction.

\section{Proof of Theorems 1.1 and 1.2}

Observe first that since the construction of the previous section does not modify the metric in a neighborhood of the soul, the set of vectors normal to $S$ is the same in the original and the final metric. Hence we only need to check that if $\gamma$ was a radial geodesic in $M$, then $\tilde{\gamma}(t)=(\gamma(t), \Gamma \circ \gamma(t))$ is a geodesic in the new metric once it is reparametrized by arc length. Writing $h(t)=\Gamma \circ \gamma(t)$ to simplify notation, it is easy to see that

$$
\nabla_{\tilde{\gamma}^{\prime}}^{P} \tilde{\gamma}^{\prime}=h^{\prime} h^{\prime \prime} \partial_{t}, \quad \nabla_{\tilde{\gamma}^{\prime}}^{N} \tilde{\gamma}^{\prime}=\frac{h^{\prime 2} h^{\prime \prime}}{\left\|\tilde{\gamma}^{\prime}\right\|^{2}} \tilde{\gamma}^{\prime}
$$

Thus after reparametrizing, $\tilde{\gamma}$ becomes a geodesic for as long as it stays in $A$. In $B$ it can be extended as $\gamma(1) \times[1, \infty)$, and therefore it is a geodesic for all its domain of definition.

Finally, thanks to the choices made at the start of section 3, we get that exp : $\nu(S) \rightarrow M$ is injective, and therefore a diffeomorphism. The part about the ideal boundary follows trivially, since clearly there is only one ray from each point not in $S$. This concludes the proof of Theorem 1.1.

Remark 4.1. The Sharafutdinov map ([6], [7]) of the metric of Theorem 1.1 is smooth, since this is the case when every normal direction from the soul is a ray.

Theorem 1.2 follows trivially by gluing together two "truncated" copies of the normal disc bundle $D(S)$ of the soul with the metrics constructed in Theorem A.

Remark 4.2. It was already known that these doubles admitted a metric with a nonnegative lower curvature bound in an Alexandrov sense: namely, we could always take the double of two sublevel sets of the Cheeger-Gromoll convex function (see [1] and [4]) However, such a metric is usually nonsmooth at the glued part. Furthermore, the spaces of directions at such points are not isometric to spheres except if the mentioned convex function has some extra regularity.

Remark 4.3. Theorem 1.2 shows that any open manifold with nonnegative curvature is diffeomorphic to the interior of a closed convex set in a compact manifold of nonnegative curvature. The converse is also true, as follows from Theorems A, 2.2, and the fact that the interior of such a set is diffeomorphic to an open tubular neighborhood of its soul.

\section{REFERENCES}

[1] Jeff Cheeger and Detlef Gromoll, On the structure of complete manifolds of nonnegative curvature, Annals of Mathematics 96 (1972), no. 3, 413-443. MR 46:8121

[2] Jose F. Escobar and Alexandre Freire, The spectrum of the Laplacian of manifolds of positive curvature, Duke Mathematical Journal 65 (1992), no. 1, 1-21. MR 93d:58174

[3] Stephen Kronwith, Convex manifolds of nonnegative curvature, Journal of Differential Geometry 14 (1979), 621-628. MR 82k:53063

[4] Grisha Perelman, Alexandrov's spaces with curvatures bounded from below, ii, Preprint.

[5] _ Proof of the soul conjecture of Cheeger and Gromoll, Journal of Differential Geometry 40 (1994), 209-212. MR 95d:53037 
[6] V. A. Sharafutdinov, The Pogorelov-Klingenberg theorem for manifolds homeomorphic to $\mathbb{R}^{n}$, Siberian Mathematical Journal 18 (1977), 915-925. MR 58:7488

[7] Jin-Whan Yim, Distance nonincreasing retraction on a complete open manifold of nonnegative sectional curvature, Ann. Global Anal. Geom. 6 (1988), no. 2, 191-206. MR 90a:53049

Department of Mathematics, University of Pennsylvania, Philadelphia, Pennsylvania 19104

E-mail address: guijarro@math.upenn.edu 